\title{
WHEN HIV CLINICIANS PREVENT SOCIAL SCIENTISTS FROM ACCESSING 'THEIR' PATIENTS: SOME ETHICAL CONCERNS
}

\author{
Nicoli Nattrass \\ AIDS and Society Research Unit, Centre for Social Science Research, University of Cape Town
}

There is an understandable tension between medical researchers and social scientists when it comes to AIDS-related research at clinic level. Clinics offering antiretroviral treatment exist primarily to assist patients - but many of them also provide 'data' for medical researchers. This may involve a randomised controlled trial, or simply the collection of data on adherence. A social scientist wishing to access patients to conduct interviews or focus groups thus (inevitably) appears to the HIV clinician as at best a disruption to an already over-stretched operation, and at worst a potentially confounding factor in his or her own research.

Given that the medical practitioner/researchers effectively control the clinics, the temptation to deny social scientists access to patients must be strong. As discussed below, this was the case with regard to two Cape Town clinics that prevented a clinical psychologist (working in my research unit) from conducting social science research. Although understandable, such unaccountable exercise of power denies patients the opportunity to participate in other research projects that may be of benefit to them (or society) in ways that HIV clinicians do not necessarily appreciate. As such, it violates the principle of informed consent. It is also problematic in that it restricts the AIDS research agenda to biomedical concerns. This is particularly worrying with regard to AIDS, where it is widely accepted (by social scientists and HIV clinicians alike) that social and psychological factors matter a great deal for HIV prevention and treatment interventions.

\section{GATE-KEEPING BY MEDICAL}

RESEARCHER/PRACTITIONERS: A CASE STUDY

The wider Cape Town metropolitan area is home to several antiretroviral treatment and vaccine initiatives funded by NGOs and pharmaceutical companies. All of these entail collaboration with the Western Cape government (which has de jure ownership of the clinics). Most of the projects have strong links with university-based medical researchers, some of whom also offer medical or related support services to the projects. Many HIV clinicians are thus simultaneously medical practitioners and researchers.

In this world of overlap between service provision and research interests, patients on antiretroviral treatment are both beneficiaries and research subjects. They enter a world entirely controlled by medical practitioner/researchers. Although the clinics are technically under the control of the government, decision-making power in effect is ceded to the largely foreign-funded doctors and researchers who run the interventions. They decide who can be on the premises, what research is 'acceptable', and who can interview the patients. This gatekeeper role has serious implications for social scientists trying to conduct research - as one of my PhD students discovered to her cost.
The student, a trained and registered clinical psychologist, wanted to conduct research into the psychological well-being and coping strategies of low socio-economic status mothers on antiretroviral treatment. Her research entailed recruiting 75 HIV-positive mothers and interviewing them when they started treatment, and then again after 4, 12 and 24 weeks. A sample of 75 HIV-negative mothers from the community was to be recruited as the comparison group. The aim of the research was to explore the challenges to women's care-giving and psychological well-being posed by negotiating the multiple roles of living with HIV, caring for young children and dealing with the general stressors associated with poverty. She was particularly interested in drawing out the implications of these multiple roles for women's adherence to antiretroviral therapy.

In addition to interviewing the women, the student stated her intention to ask the participants for permission to access their medical files so that she could collect data on CD4 counts, viral loads, clinical staging and adherence information. She stressed that this information would only be collected at the convenience of the clinic. In her research protocol, she acknowledged that she would need the help of clinic staff in accessing the files, but argued that this cost was small in relation to the benefits of her study. She pointed out that she and her researchers are clinically trained (and registered with 
the Health Professions and Social Work Councils) and thus in a position to provide useful feedback to the clinic on patients who were deemed to be at risk for poor adherence owing to their mental health and social difficulties. She also pointed out that some of the empirical indicators being developed in her study could potentially become useful tools for the counsellors associated with the treatment programme to use in the future should they wish to assess the extent to which a patient's psychological well-being places them at risk for poor adherence: 'More broadly, the research aims to make recommendations about the kinds of psychosocial services which can enhance women patients' quality of life and psychological well-being, as well as enhancing their adherence to treatment' (student research protocol).

The student approached a clinic in a local African township for permission to invite potential respondents to participate in her study. The medical practitioner/researchers associated with the antiretroviral treatment project at the clinic considered her request - but rejected it. As can be seen from the three reasons listed below, the fact that the research and treatment intervention was funded in part through a large pharmaceutical company seemed to pose particular problems.

1. 'The population is over-researched and your study is not the primary focus of the research' ('the research' referring, of course, to the research already being conducted by the medical doctors/researchers linked to the clinic);

2. 'None of the (pharmaceutical company) Exco members are senior authors of the project' (i.e. the student's doctoral project); and

3. 'It has not been approved by the same Ethics Committee as the other (pharmaceutical company) projects' (written response to the student).

The student was then referred to another clinic in a different African township, but was again turned down by the gatekeeper committee of medical practitioner/researchers. This time she was turned down principally because:

1. There were insufficient 'direct and tangible benefits to the clinic patients';

2. They were concerned about the 'amount of time' respondents would have to spend on the study; and

3. They did not 'feel that the research addresses the needs which they have as a clinic at this point in time, and only want to permit research which does so' (response to the student).

The student responded to the main research gatekeeper of this clinic by reiterating that her research could be of potential direct and immediate benefit to the counsellors who work with people on antiretroviral treatment as well as to the patients themselves: 'Depending on the clinic's need, feedback from the research could either be restricted to liaising with the counselling team, or direct input could also be given to the other members of the clinic team if relevant and useful. Whichever approach minimises the negative impact of our presence on the running of the service, while maximising the support and benefits, is desirable for me' (response by the student).

She never got a reply to this correspondence. She made several phone calls (all to no effect) and eventually gave up. Fortunately, a local clinic run by Absolute Return for Kids (ARK) came to the rescue and let her conduct her research there. The fact that it was not linked to either a pharmaceutical company or a major research project no doubt helped ...

This story of her rejection by the first two clinics is interesting in several respects. Firstly, with regard to both clinics she approached, the medical practitioner/researchers prioritised their own research interests and used their effective control over the clinic to prevent other research from taking place. The blocking of research by the first clinic on the grounds that only research linked to the pharmaceutical company that was funding it would be acceptable was breath-taking in its blatant self-interest.

Secondly, it is clear from the reasons provided by the second clinic that non-medical interventions were not regarded as potentially being of value to the patient. This reflects an uncritical adoption of a strictly biomedical notion of health promotion which is particularly worrying given the obvious social and psychological determinants of adherence to antiretroviral therapy. It also demonstrates a lack of understanding that even when the 'benefits' of social science research for a particular individual patient are not obvious, the research may nevertheless inform the development of relevant future social policies. A narrow application of the requirement that the research 'benefits' the research subject is therefore necessarily always going to be biased against the social scientist.

Thirdly, the story demonstrates a paternalistic approach on the part of medical practitioner/researchers to who is in the best position to judge whether a project is, or is not, in the best interests of the patient. This, in my view, runs counter to the spirit of the principle of informed consent. Although the principle of informed consent was designed to ensure that prospective research subjects have the right to refuse to participate, it surely also protects these same subjects from others deciding on their behalf that it is not in their best interests to participate. The principle should surely be that research subjects have the right to decide whether they want - or do not want - to participate. In my experience of conducting surveys and qualitative in-depth interviews, research subjects often enjoy being interviewed and having the opportunity to discuss matters of concern to them (see also Pahl'). This is, of course, not true for all participants. Some get irritated by the research process, but they can always refuse to participate at any point (as is typically - if not always - pointed out in consent forms).

Ultimately, it must be up to the potential research participant to decide whether the risks (which, in social science research, typically entail little more than the opportunity cost of the time taken up by the interview) outweigh the benefits. Medical 
practitioner/researchers are not in an appropriately informed position to make the judgement call.

\section{SOME ETHICAL CONCERNS}

Most research ethics codes are silent on the issue of ensuring that potential research subjects have the right to choose whether they do or do not want to participate in research. The European Group on Ethics in Science and New Technologies ${ }^{2}$ and the Nuffield Council on Bioethics (UK) note that in some local contexts, it may be appropriate to obtain family- or community-level agreement before approaching research subjects (see discussion in $\mathrm{NCOB}_{1}{ }^{4}$ p. 73). However, this is a concession to local culture - it cannot be used to justify giving medical practitioner/researchers the right to make decisions on the behalf of their patients, especially in cases where these same medical practitioner/researchers are not disinterested observers.

Before 1947, when the Nuremburg code of ethics for medical research highlighted the need for informed consent, ${ }_{1}^{5}$ the prevailing 'Hippocratic' approach assumed that medical researchers and doctors were the only agents capable of making appropriate judgements about medical research. ${ }^{6}$ Refusing to allow patients on antiretroviral therapy the opportunity to make their own decision as to whether they wish to participate in a psychological study or not amounts to a reversion to this old paternalistic approach.

Some attention has been paid to the problem of 'dual loyalty' - i.e. when a clinician experiences a role conflict between their professional duties to a patient and the obligations, expressed or implied, real or perceived, to the interests of a third party (see Physicians for Human Rights and University of Cape Town Health Sciences Faculty ${ }^{7}$ ). However, as Singh points out, ${ }_{1}^{8}$ this problem has yet to be applied to dual loyalty in medical research - especially when the roles of clinician and researcher merge: 'In the practice-research context this translates to the physician-researcher's primary interest (duty of care towards the patient-subject) being undermined by secondary factors (such as loyalty to the study/sponsor)' (p. 395). Furthermore, clinicians may deny social scientists access to 'their' patients not because they are worried about the adverse implications of the social science research for the patients, but because they do not want any other research (besides their own) being conducted on the patients.
Disciplinary differences like this would not matter except for the fact that medical practitioner/researchers are in a powerful position to dictate the research agenda largely because they have access to extraordinarily large budgets to treat and research their patients. In a very real sense, these medical practitioner/researchers do indeed control 'their' patients/research subjects - and as discussed above, they can use this power and deny others research access to them.

With about a fifth of the adult population HIV-positive, and given the government's reluctance to roll out antiretroviral therapy with any sense of urgency, there is substantial pressure (both in terms of resources and emotional energies) on HIV clinicians. Their irritation with social science research is therefore in some way understandable. However, as the AIDS crisis is both a social and health crisis, and given that social scientists are often better placed than medical practitioners to understand the social and behavioural context governing individual adherence to antiretroviral therapy, the effective control of medical practitioner/researchers over access to research subjects is highly problematic. There needs to be a more tolerant and constructive attitude towards social science research on the part of HIV clinicians who control access to patients on antiretroviral therapy.

\section{REFERENCES}

1. Pahl J. Ethics review in social care research. Paper presented at the Emerging Ethical Issues in Social Science and Cross-Cultural Research, University of Sussex, Brighton, May 2005.

2. European Group on Ethics in Science and New Technologies (EGE). Opinion Nr 17 on the Ethical Aspects of Clinical Research in Developing Countries. 2003. Available on http://europa.eu.int/comm./European_group_ethics/docs/ avis17_en.pdf.

3. Nuffield Council on Bioethics (NCOB). The ethics of research related to healthcare in developing countries. April 2002. Available on http://www. nuffieldbioethics.org/developingcountries.

4. Nuffield Council on Bioethics (NCOB). The ethics of research related to healthcare in developing countries: A follow-up Discussion Paper based on a workshop held in Cape Town, South Africa, 12 - 14 February, 2004. 2005. Nuffield Council on Bioethics, London. See also http://www.nuffieldbioethics. org

5. Hoeyer K, Dahlager L, Lynoe N. Conflicting notions of research ethics: The mutually challenging traditions of social scientists and medical researchers. Soc Sci Med (in press).

6. Alderson P. Ethical codes in social science and medicine. Paper presented at the Emerging Ethical Issues in Social Science and Cross-Cultural Research, University of Sussex, Brighton, May 2005.

7. Physicians for Human Rights and University of Cape Town Health Sciences Faculty. Dual loyalty and human rights in health professional practice: Proposed Guidelines and Institutional Standards. 2003. Available on http://www. phrusa.org/healthrights/dual_loyalty.html.

8. Singh J. The impact on ethics. In: Karim S, Karim Q, eds. HIV/AIDS in South Africa. Cape Town: Cambridge University Press, 2005: 384-404. 